\title{
A Case of Amelanotic Melanoma in the Setting of Cemiplimab Therapy for Invasive Squamous Cell Carcinoma
}

\author{
Victoria Lee, $\mathrm{PhD}^{1}$, Mark D. Hoffman, $\mathrm{MD}^{2}$, Arlene M. Ruiz de Luzuriaga, MD, MPH² \\ ${ }^{1}$ Pritzker School of Medicine, University of Chicago, Chicago, IL \\ 2 Section of Dermatology, Department of Medicine, University of Chicago, Chicago, IL
}

\section{ABSTRACT}

Cemiplimab is the first PD-1 inhibitor approved in the United States for the treatment of metastatic or locally advanced cutaneous squamous cell carcinoma (SCC) and basal cell carcinoma (BCC), but not melanoma. We describe a case of amelanotic melanoma (AM) developing in a patient on cemiplimab therapy for invasive SCC. Cutaneous AM is a rare subtype of cutaneous melanoma that shows little or no melanin pigmentation on clinical and histological examination thus often leading to a high misdiagnosis rate. Physicians should consider atypical presentations of melanoma, including amelanotic melanoma, during surveillance of patients managed with immune checkpoint inhibitor therapy.

\section{INTRODUCTION}

Cemiplimab is a monoclonal antibody directed against programmed-death receptor 1 (PD-1). It is the first PD-1 inhibitor approved in the United States for the treatment of metastatic or locally advanced cutaneous squamous cell carcinoma (SCC) and basal cell carcinoma (BCC). ${ }^{1-4}$ Although other PD-1 inhibitors have assumed important roles in the management of melanoma, cemiplimab does not currently have this indication. We describe a case of amelanotic melanoma developing in a patient on cemiplimab therapy for invasive SCC. Due to insufficient tyrosinase activity or quantity, cutaneous amelanotic melanoma (AM) shows little or no melanin pigmentation on clinical and histological examination thus often leading to a high misdiagnosis rate. ${ }^{5}$ Several studies have shown an association of AMs with high mitotic and growth rates, and with higher prevalence of vertical growth phase and greater Breslow scale at diagnosis than pigmented melanomas (PMs), suggestive of an intrinsically aggressive phenotype. ${ }^{6,7}$ Consequently, it is frequently diagnosed at advanced stages.

\section{CASE REPORT}

A 72-year-old man presented for skin cancer surveillance. He had a history of basosquamous carcinoma (BSC) of the scalp with extension into the calvarium diagnosed 2.5 years earlier, which progressed following surgical resection and chemoradiotherapy. He had a complete response to 35 cycles of cemiplimab therapy over two years, and treatment was discontinued one month prior to presentation. On examination, a $6 \mathrm{~mm}$ pink papule was noted on the right arm (Figure 
1). The patient believed this lesion had appeared in the past one to two months; his previous skin cancer surveillance had been 19 months earlier. The clinical concern was for BCC.

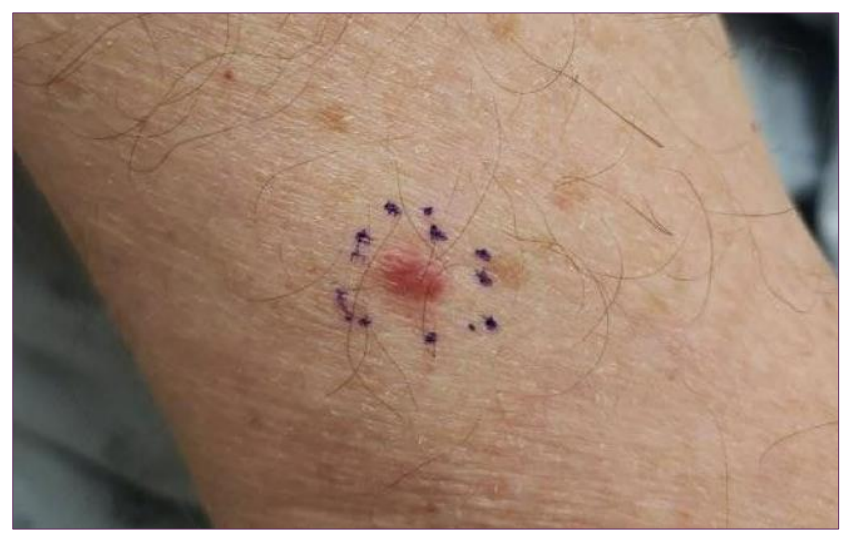

Figure 1. A $6 \mathrm{~mm}$ pink papule noted on the right upper arm.

Histopathologic study of the shave biopsy led to a final diagnosis of malignant melanoma (Clark Level IV, Breslow thickness at least $1 \mathrm{~mm}$ ) with positive lateral and focally deep margins. The lesion was not heavily melanized (Figures $2 \mathrm{~A}$ and $2 \mathrm{~B}$ ). PD-L1 expression within the melanocytic lesion was high ( $>5 \%$ of tumor cells), with a heterogeneous expression pattern (Figure 2C). Brisk CD8+ tumor-infiltrating lymphocytes were observed in geographic association with high PD-L1 expression (Figure 2D). The patient underwent wide local excision and sentinel lymph node biopsy. Final pathology showed a residual melanoma in-situ component, with negative deep and lateral margins, and negative sentinel lymph nodes.

\section{DISCUSSION}

Unlike other PD-1 inhibitors in its class, cemiplimab is not presently indicated for the treatment of patients with advanced melanoma, though clinical trials of combination therapy with cemiplimab for this indication are currently underway. ${ }^{8}$ In our patient it appears that the AM lesion arose during and near the completion of cemiplimab therapy. Given that some immune checkpoint inhibitors are considered first-line therapy for unresectable or metastatic melanoma, it is striking that this melanoma was found in our patient after two years of treatment with a PD- 1 inhibitor, and that it was clinically amelanotic and histologically poorly melanized.

While amelanosis is not a feature known to be associated with resistance to PD-1 inhibitor therapy to date, vitiligo-like depigmentation has been associated with PD-1 inhibitors in the context of melanoma management, and this development has been linked to improved outcomes. ${ }^{9}$ Interestingly, recognition of tyrosinase by tumor-infiltrating lymphocytes has been demonstrated in a melanoma patient responding to immunotherapy. ${ }^{10}$ As it is known that the lack of melanin pigmentation in $A M$ is due to insufficient tyrosinase activity or quantity, ${ }^{5}$ it is possible that the lesion observed in our patient progressed because it lacked a potential antigen for immune recognition. Alternatively, it is also possible that recognition of tyrosinase by tumorinfiltrating lymphocytes during the course of receiving immunotherapy led to the destruction of tyrosinase-active melanized cells, thereby leaving behind an amelanotic lesion. 


\section{SKIN}

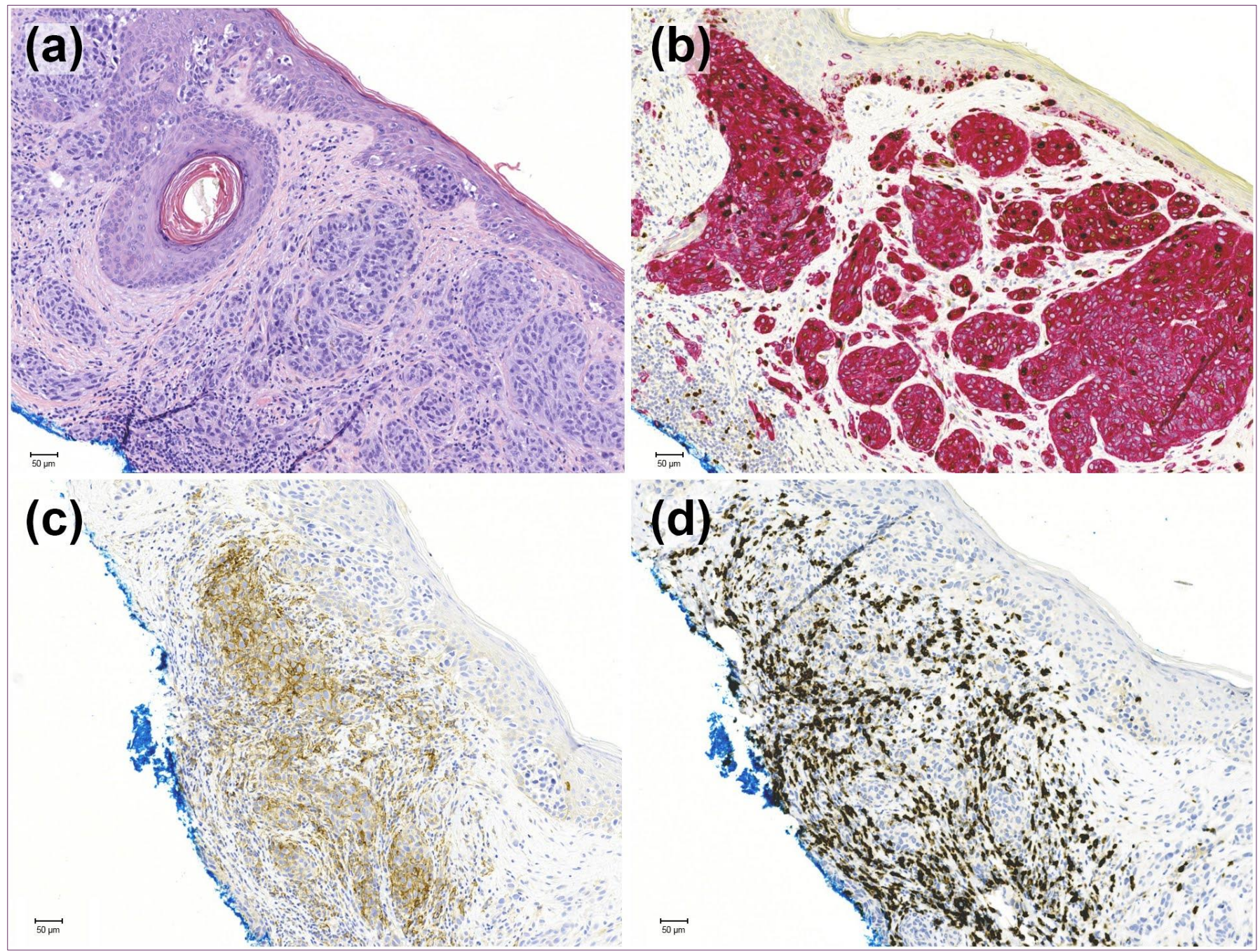

Figure 2. A) $\times 10 \mathrm{H} \& \mathrm{E}$ staining showing proliferation of poorly melanized melanocytes with severe cytologic atypia extending into the dermis, and mitotic figures up to 7 per $1 \mathrm{~mm}^{2} \quad$ B) $\times 10$ immunohistochemical (IHC) staining with Melan-A/Ki67 showing pagetoid spread of melanocytes and increased proliferative activity within the dermis C) $\times 10$ IHC staining with PD-L1 showing high expression within the melanocytic lesion ( $>5 \%$ of tumor cells) with heterogeneous expression pattern D) $\times 10 \mathrm{IHC}$ staining with CD8 showing brisk CD8+ tumor-infiltrating lymphocytes in geographic association with high PD-L1 expression.

\section{CONCLUSION}

The reliability of intratumoral PD-L1 expression as a predictive biomarker of response to PD-1/PD-L1 inhibitor therapy remains controversial. ${ }^{11,12}$ PD-L1 expression levels and patterns have been shown to vary across melanoma subtypes and morphology, and are often geographically associated with CD8+ lymphocytes, similar to our case. ${ }^{12}$ Physicians should consider atypical presentations of melanoma, including amelanotic melanoma, during surveillance of patients managed with immune checkpoint inhibitor therapy.

Conflict of Interest Disclosures: None

Funding: None

Corresponding Author:

Arlene M. Ruiz de Luzuriaga, MD, MPH

5841 S Maryland Ave, MC 5067

Chicago, IL 60637

Email: aruizde@medicine.bsd.uchicago.edu 


\section{References:}

1. Migden MR, Rischin D, Schmults CD, et al. PD-1 blockade with cemiplimab in advanced cutaneous squamous-cell carcinoma. N Engl J Med 2018; 379(4):341-351.

2. Migden MR, Khushalani NI, Chang ALS, et al. Cemiplimab in locally advanced cutaneous squamous cell carcinoma: results from an openlabel, phase 2, single-arm trial. Lancet Oncol 2020; 21(2):294-305.

3. Ahmed SR, Petersen E, Patel R, et al. Cemiplimab-rwlc as first and only treatment for advanced cutaneous squamous cell carcinoma. Expert Rev Clin Pharmacol 2019; 12(10):947951.

4. Stratigos AJ, Sekulic A, Perris K, et al. Cemiplimab in locally advanced basal cell carcinoma after hedgehog inhibitor therapy: an open-label, multi-centre, single-arm, phase 2 trial. Lancet Oncol 2021; S1470-2045(21):00126-1.

5. Gong H, Zheng H, Li J. Amelanotic melanoma. Melanoma Res 2019; 29(3):221-230.

6. Thomas NE, Kricker A, Waxweiler WT, et al. Comparison of clinicopathologic features and survival of histopathologically amelanotic and pigmented melanomas: a population-based study. JAMA Dermatol 2014; 150(12):13061314.

7. McClain SE, Mayo KB, Shada AL, et al. Amelanotic melanomas presenting as red skin lesions: a diagnostic challenge with potentially lethal consequences. Int J Dermatol 2012; 51(4):420-426.

8. Clinical Trials Using Cemiplimab [Internet]. National Cancer Institute; n.d. [cited 2021 Jun 2]. Available from: https://www.cancer.gov/aboutcancer/treatment/clinicaltrials/intervention/cemiplimab

9. Teulings $\mathrm{H}$, Limpens J, Jansen SN, et al. Vitiligolike depigmentation in patients with stage III-IV melanoma receiving immunotherapy and its association with survival: a systematic review and meta-analysis. J Clin Oncol 2015; 33(7):773-781.

10. Robbins PF, El-Gamil M, Kawakami Y, et al. Recognition of tyrosinase by tumor-infiltrating lymphocytes from a patient responding to immunotherapy. Cancer Res 1994; 54:31243126.

11. Long GV, Larkin J, Ascierto PA, et al. PD-L1 expression as a biomarker for nivolumab (NIVO) plus ipilimumab (IPI) and NIVO alone in advanced melanoma (MEL): a pooled analysis. Ann Oncol 2016; 27(6_suppl):1112PD-PD.
12. Kaunitz GJ, Cottrell TR, Lilo M, et al. Melanoma subtypes demonstrate distinct PD-L1 expression profiles. Laboratory Investigation 2017; 97:10631071. 
